\title{
Efficient Image Reconstruction for Gigapixel Quantum Image Sensors
}

\section{Citation}

Chan, Stanley H., and Yue M. Lu. 2014. “Efficient Image Reconstruction for Gigapixel Quantum Image Sensors." In Proceedings of the IEEE Global Conference on Signal and Information Processing (GlobalSIP) 2014, Atlanta, GA, December 3-5.

\section{Permanent link}

http://nrs.harvard.edu/urn-3:HUL.InstRepos:25482689

\section{Terms of Use}

This article was downloaded from Harvard University's DASH repository, and is made available under the terms and conditions applicable to Open Access Policy Articles, as set forth at http:// nrs.harvard.edu/urn-3:HUL.InstRepos:dash.current.terms-of-use\#OAP

\section{Share Your Story}

The Harvard community has made this article openly available.

Please share how this access benefits you. Submit a story.

Accessibility 


\title{
EFFICIENT IMAGE RECONSTRUCTION FOR GIGAPIXEL QUANTUM IMAGE SENSORS
}

\author{
Stanley H. Chan and Yue M. Lu \\ School of Engineering and Applied Sciences \\ Harvard University, Cambridge, MA 02138, USA \\ Email:\{schan, yuelu\}@ seas.harvard.edu
}

\begin{abstract}
Recent advances in materials, devices and fabrication technologies have motivated a strong momentum in developing solid-state sensors that can detect individual photons in space and time. It has been envisioned that such sensors can eventually achieve very high spatial resolutions (e.g., $10^{9}$ pixels/chip) as well as high frame rates (e.g., $10^{6}$ frames/sec). In this paper, we present an efficient algorithm to reconstruct images from the massive binary bit-streams generated by these sensors. Based on the concept of alternating direction method of multipliers (ADMM), we transform the computationally intensive optimization problem into a sequence of subproblems, each of which has efficient implementations in the form of polyphase-domain filtering or pixel-wise nonlinear mappings. Moreover, we reformulate the original maximum likelihood estimation as maximum a posterior estimation by introducing a total variation prior. Numerical results demonstrate the strong performance of the proposed method, which achieves several dB's of improvement in PSNR and requires a shorter runtime as compared to standard gradient-based approaches.
\end{abstract}

Index Terms - Image reconstruction, quantum image sensors, gigapixel imaging, ADMM

\section{INTRODUCTION}

Thanks to recent advances in materials and fabrication technologies, there has been an emerging class of solid-state imaging sensors that are capable of achieving single-photon sensitivity, sub-nanosecond time resolution and rapidly increasing spatial resolutions [1,2]. These devices, collectively referred to as quantum image sensors (QIS) in this work, are envisioned as the next generation imaging technology after CMOS [3], with numerous applications [4-7].

The operational principle of a QIS is analogous to photographic film: Photon flux reaching a pixel of the single-photon detector triggers a binary response, thus generates a 1-bit signal revealing the intensity of the flux during the exposure. With high frame rates (e.g., $10^{6}$ frames/sec) and increasing spatial resolutions (e.g., $10^{9}$ pixels/chip as envisioned in [3]), the QIS generates a massive stream of bits, which must be "decoded" to recover the underlying image.

The 1-bit measurements acquired by the QIS follow a quantized Poisson process. Therefore, image reconstruction from these measurements can be solved via maximum likelihood estimation (MLE). However, except for simple cases where the photon flux is a piecewise constant function, the MLE does not have a closed-form solution in general, even though the problem is convex [8]. Iterative algorithms have been proposed, e.g., [9-11], but how to improve the slow convergence is still an open problem.

The work is supported, in part, by the U.S. National Science Foundation under Grant CCF-1319140.
By recognizing the separable structure of the MLE's objective function, we propose a new algorithm based on the concept of alternating direction method of multipliers (ADMM) $[12,13]$. The contributions of this paper are two-fold. First, we present fast solutions to the subproblems associated with the ADMM formulation. One key technique we use is the polyphase representation, a powerful tool from multirate signal processing [14]. Second, we introduce a total variation (TV) prior and modify the MLE problem to a maximum a posterior (MAP) problem. We show empirically that the MAP formulation significantly improves the reconstruction quality with only marginal increase in computational cost.

The rest of the paper is organized as follows. We first present the modeling of QIS and elaborate on the MLE problem in Section 2. In Section 3 we discuss the proposed ADMM algorithm. Experimental results are shown in Section 4 and we conclude in Section 5.

\section{MODEL AND PROBLEM FORMULATION}

The signal processing model of a QIS sensor consists of two major components: (1) The light exposure stage which models the propagation of light from scene to sensor; (ii) The sensing stage which models the conversion of the light field to the binary measurements. A pictorial illustration is shown in Figure 1, and each component is described as follows.

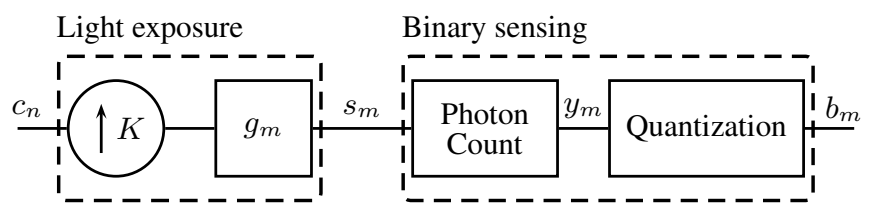

Fig. 1: The two mathematical components of modeling the image formation of QIS (c.f. [10]).

\subsection{Modeling of Light Exposure}

Consider a light field intensity $\lambda(x)$ of spatial coordinate $x$. Without loss of generality we shall assume that $\lambda(x)$ is a one-dimensional function and that the support of the coordinate is the unit interval, i.e., $0 \leq x \leq 1$. Furthermore, due to the presence of diffraction limit of the lens, we model $\lambda(x)$ as a function in the shift-invariant space spanned by a non-negative interpolation kernel $\varphi(x)$ :

$$
\lambda(x)=\frac{N}{\tau} \sum_{n=0}^{N-1} c_{n} \varphi(N x-n),
$$

where $\tau$ is the exposure time, $\left\{c_{n}: c_{n} \geq 0\right\}$ is a set of variables encoding the scene, and $N$ is the number of variables. The constant $N / \tau$ is not essential, but it simplifies the analysis below. 
Suppose that the sensor consists of $M$ pixels per unit space and that the $m$ th pixel covers the area $[m / M,(m+1) / M]$, then the total light exposure accumulated on the surface area of the $m$ th pixel within a time period $[0, \tau]$ is

$$
s_{m} \stackrel{\text { def }}{=} \int_{0}^{\tau} \int_{m / M}^{(m+1) / M} \lambda(x) d x d t=\tau\langle\lambda(x), \beta(M x-m)\rangle,
$$

where $\langle\cdot, \cdot\rangle$ represents the standard $L^{2}$ inner product, and $\beta(x)$ is the box function defined as $\beta(x)=1$ for $0 \leq x \leq 1$ and is zero otherwise. Substituting (1) into (2) yields

$$
s_{m}=\sum_{n=0}^{N-1} c_{n} g_{m-K n}
$$

where $K \stackrel{\text { def }}{=} M / N$ is the spatial oversampling ratio, and

$$
g_{m} \stackrel{\text { def }}{=}\langle\varphi(x), \beta(K x-m)\rangle
$$

is the $m$ th coefficient of a discrete filter. Using matrix-vector notations, the expression in (3) can be equivalently written as $\boldsymbol{s}=\boldsymbol{G} \boldsymbol{c}$, for appropriate $s \in \mathbb{R}^{M \times 1}, \boldsymbol{c} \in \mathbb{R}^{N \times 1}$ and $\boldsymbol{G} \in \mathbb{R}^{M \times N}$.

\subsection{Modeling of Binary Sensing}

Let $y_{m}$ be the number of photons impinging on the surface of the $m$ th pixel during an exposure period $[0, \tau]$. We model the relationship between $s_{m}$ and $y_{m}$ as a Poisson process $Y_{m}$ :

$$
\mathbb{P}\left[Y_{m}=y_{m} ; s_{m}\right]=\frac{s_{m}^{y_{m}} e^{-s_{m}}}{y_{m} !}
$$

The binary sensing of the QIS quantizes the observed photon count $y_{m}$ through a threshold operation. In this paper, we assume that the threshold level is set to unity so that the binary response is

$$
B_{m}=1 \text { if } Y_{m} \geq 1 \text { and } B_{m}=0 \text { if } Y_{m}=0 .
$$

Hence, the probability of observing $B_{m}$ is

$$
\mathbb{P}\left[B_{m}=1\right]=1-e^{-s_{m}} \quad \text { and } \quad \mathbb{P}\left[B_{m}=0\right]=e^{-s_{m}} .
$$

\subsection{Maximum Likelihood Estimation}

The image reconstruction task of the QIS can be formulated as the following maximum likelihood estimation (MLE):

$$
\begin{aligned}
\boldsymbol{c}^{*} & =\underset{\boldsymbol{s}=\boldsymbol{G} \boldsymbol{c}}{\arg \max } \prod_{m=1}^{M} \mathbb{P}\left[B_{m}=b_{m} ; s_{m}\right] \\
& =\underset{\boldsymbol{s}=\boldsymbol{G} \boldsymbol{c}}{\arg \min }-\sum_{m=1}^{M} \log \left(\left(1-b_{m}\right) e^{-s_{m}}+b_{m}\left(1-e^{-s_{m}}\right)\right)
\end{aligned}
$$

Defining $F(\boldsymbol{s}) \stackrel{\text { def }}{=}-\sum_{m=1}^{M} \log \left(\left(1-b_{m}\right) e^{-s_{m}}+b_{m}\left(1-e^{-s_{m}}\right)\right)$ and introducing a prior $p(\boldsymbol{c})$, we can further modify the MLE to a maximum a posterior (MAP) estimation problem:

$$
\boldsymbol{c}^{*}=\underset{\boldsymbol{s}=\boldsymbol{G} \boldsymbol{c}}{\arg \min } F(\boldsymbol{s})-\log p(\boldsymbol{c}) .
$$

In this paper, we consider the total variation prior

$$
p(\boldsymbol{c}) \propto \exp \left(-\lambda\|\boldsymbol{c}\|_{T V}\right)
$$

where $\|\boldsymbol{c}\|_{T V}=\|\boldsymbol{D} \boldsymbol{c}\|_{1}$ and $\boldsymbol{D}$ is the first order finite difference operator. Thus, (9) is equivalent to

$$
\underset{\boldsymbol{c}}{\operatorname{minimize}} F(\boldsymbol{s})+\lambda\|\boldsymbol{D} \boldsymbol{c}\|_{1}, \quad \text { subject to } \boldsymbol{s}=\boldsymbol{G} \boldsymbol{c},
$$

which is a convex optimization problem. In the following sections we present an efficient algorithm to solve (11).

\section{IMAGE RECONSTRUCTION ALGORITHM}

The proposed image reconstruction algorithm is based on the concept of alternating direction method of multipliers (ADMM) [13]. The idea is to consider the following equivalent constrained problem

$$
\underset{c}{\operatorname{minimize}} F(\boldsymbol{s})+\lambda\|\boldsymbol{v}\|_{1}, \quad \text { subject to } \boldsymbol{s}=\boldsymbol{G} \boldsymbol{c}, \boldsymbol{v}=\boldsymbol{D} \boldsymbol{c}
$$

by defining an auxiliary variable $\boldsymbol{v} \in \mathbb{R}^{N \times 1}$. Then, we consider the augmented Lagrangian function:

$$
\begin{aligned}
\mathcal{L}(\boldsymbol{c}, \boldsymbol{s}, \boldsymbol{v}, \boldsymbol{z}, \boldsymbol{r}) \stackrel{\text { def }}{=} F(\boldsymbol{s}) & +\lambda\|\boldsymbol{v}\|_{1}-\boldsymbol{z}^{T}(\boldsymbol{s}-\boldsymbol{G} \boldsymbol{c})+\frac{\rho}{2}\|\boldsymbol{s}-\boldsymbol{G} \boldsymbol{c}\|^{2} \\
& -\boldsymbol{r}^{T}(\boldsymbol{v}-\boldsymbol{D} \boldsymbol{c})+\frac{\gamma}{2}\|\boldsymbol{v}-\boldsymbol{D} \boldsymbol{c}\|^{2}
\end{aligned}
$$

where $\boldsymbol{z} \in \mathbb{R}^{M \times 1}$ and $\boldsymbol{r} \in \mathbb{R}^{N \times 1}$ are the Lagrange multipliers associated with the constraints $\boldsymbol{s}=\boldsymbol{G} \boldsymbol{c}$ and $\boldsymbol{v}=\boldsymbol{D} \boldsymbol{c}$, respectively, and $\rho>0$ and $\gamma>0$ are parameters that control the emphasis of the quadratic penalty functions. ADMM states that the saddle point of $\mathcal{L}$ is the optimal solution of (12). Consequently, (12) can be solved by alternatingly solving the following sequence of subproblems:

$$
\begin{aligned}
& \boldsymbol{c}^{(k+1)}=\underset{\boldsymbol{c}}{\arg \min } \mathcal{L}\left(\boldsymbol{c}, \boldsymbol{s}^{(k)}, \boldsymbol{v}^{(k)}, \boldsymbol{z}^{(k)}, \boldsymbol{r}^{(k)}\right), \\
& \boldsymbol{s}^{(k+1)}=\underset{\boldsymbol{s}}{\arg \min } \mathcal{L}\left(\boldsymbol{c}^{(k+1)}, \boldsymbol{s}, \boldsymbol{v}^{(k)}, \boldsymbol{z}^{(k)}, \boldsymbol{r}^{(k)}\right), \\
& \boldsymbol{v}^{(k+1)}=\underset{\boldsymbol{v}}{\arg \min } \mathcal{L}\left(\boldsymbol{c}^{(k+1)}, \boldsymbol{s}^{(k+1)}, \boldsymbol{v}, \boldsymbol{z}^{(k)}, \boldsymbol{r}^{(k)}\right), \\
& \boldsymbol{z}^{(k+1)}=\boldsymbol{z}^{(k)}-\rho\left(\boldsymbol{s}^{(k+1)}-\boldsymbol{G} \boldsymbol{c}^{(k+1)}\right), \\
& \boldsymbol{r}^{(k+1)}=\boldsymbol{r}^{(k)}-\gamma\left(\boldsymbol{v}^{(k+1)}-\boldsymbol{D} \boldsymbol{c}^{(k+1)}\right) .
\end{aligned}
$$

We now discuss how each of the above subproblems is solved. For notational simplicity we drop the iteration number $(\cdot)^{(k)}$.

\section{1. $c$-Subproblem}

The $c$-subproblem is formulated by dropping terms independent of $c$ in (14a), yielding

$$
\underset{\boldsymbol{c}}{\operatorname{minimize}} \boldsymbol{z}^{T} \boldsymbol{G} \boldsymbol{c}+\boldsymbol{r}^{T} \boldsymbol{D} \boldsymbol{c}+\frac{\rho}{2}\|\boldsymbol{s}-\boldsymbol{G} \boldsymbol{c}\|^{2}+\frac{\gamma}{2}\|\boldsymbol{v}-\boldsymbol{D} \boldsymbol{c}\|^{2} .
$$

Setting the first order derivative to zero, the solution of (15) is

$\boldsymbol{c}=\mathcal{P}_{\mathcal{D}}\left[\left(\rho \boldsymbol{G}^{T} \boldsymbol{G}+\gamma \boldsymbol{D}^{T} \boldsymbol{D}\right)^{-1}\left(\boldsymbol{G}^{T}(\rho \boldsymbol{s}-\boldsymbol{z})+\boldsymbol{D}^{T}(\gamma \boldsymbol{v}-\boldsymbol{r})\right)\right]$

where $\mathcal{P}_{\mathcal{D}}$ is the projection onto the search domain $\mathcal{D} \stackrel{\text { def }}{=}[0,1]^{N}$. We apply $\mathcal{P}_{\mathcal{D}}$ to ensure that all estimations of $\boldsymbol{c}$ lie in $\mathcal{D}$.

The challenge of (16) is the matrix inverse. By construction, $\boldsymbol{D}^{T} \boldsymbol{D}$ is a circulant matrix and hence it is diagonalizable using the discrete Fourier transform (DFT). Thus it remains to show that $\boldsymbol{G}^{T} \boldsymbol{G}$ is also a circulant matrix, for then the matrix inverse can be implemented efficiently in the Fourier domain. 
To show that $\boldsymbol{G}^{T} \boldsymbol{G}$ is circulant, it suffices to show that $\boldsymbol{G}^{T} \boldsymbol{G}$ is a convolution matrix representing a finite impulse response (FIR) filter. Expressing the operations of $\boldsymbol{G}$ and $\boldsymbol{G}^{T}$ explicitly, we note that $\boldsymbol{G}$ is equivalent to applying an up-sampling operation followed by an FIR filter $\left\{g_{m}\right\}$, where as $\boldsymbol{G}^{T}$ is equivalent to applying the time reversal filter $\left\{g_{-m}\right\}$ followed by a down-sampling operation. The concatenation of these operations is the system shown in the middle of Figure 2.

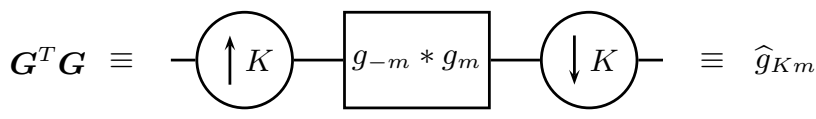

Fig. 2: The equivalence between the matrix operation $\boldsymbol{G}^{T} \boldsymbol{G}$, the system, and the finite impulse response filter $\widehat{g}_{K m}$.

To simplify the system shown in Figure 2, we denote

$$
\widehat{g}_{m} \stackrel{\text { def }}{=} g_{-m} * g_{m}
$$

and split $\widehat{g}_{m}$ into $K$ nonoverlapping polyphase components $\widehat{g}_{0, m}$, $\widehat{g}_{1, m}, \ldots, \widehat{g}_{K-1, m}$, defined as

$$
\widehat{g}_{k, m}=\widehat{g}_{K m+k}, \quad \text { for } 0 \leq k<K .
$$

Then, by using the $z$-transform and multirate identities [14] one can show the following proposition.

Proposition 1. $\boldsymbol{G}^{T} \boldsymbol{G}$ is a single convolution matrix whose impulse response is equal to $\widehat{g}_{K m}$, where $\widehat{g}_{m}$ is defined in (17).

The implication of Proposition 1 is that the matrix $\boldsymbol{G}^{T} \boldsymbol{G}$ has a circulant structure given by the FIR filter $\widehat{g}_{K m}$. This suggests that diagonalizing $\boldsymbol{G}^{T} \boldsymbol{G}$ is equivalent to applying DFT to $\left\{\widehat{g}_{K m}\right\}$, which can be done offline as $\left\{\widehat{g}_{K m}\right\}$ is fully specified by the interpolation kernel $\varphi(x)$ through (4) and (17).

\section{2. $s$-Subproblem}

Letting $\boldsymbol{d}=\boldsymbol{G} \boldsymbol{c}$ in (14b) and eliminating terms independent of $\boldsymbol{s}$, the $s$-subproblem can be written as

$$
\underset{s}{\operatorname{minimize}} F(\boldsymbol{s})-\boldsymbol{z}^{T} \boldsymbol{s}+\frac{\rho}{2}\|\boldsymbol{s}-\boldsymbol{d}\|^{2} .
$$

Since $F(s)$ is separable, we express (19) as

$$
\begin{aligned}
\underset{s}{\operatorname{minimize}} \sum_{m=1}^{M} & {\left[-\log \left(\left(1-b_{m}\right) e^{-s_{m}}+b_{m}\left(1-e^{-s_{m}}\right)\right)\right.} \\
& \left.-z_{m} s_{m}+\frac{\rho}{2}\left(s_{m}-d_{m}\right)^{2}\right]
\end{aligned}
$$

Thus, (20) is minimized if each of individual terms in the sum is minimized. Considering the $m$ th term of (20), we need to solve two cases:

$$
\begin{aligned}
& \text { if } b_{m}=0: \min _{s_{m}} s_{m}-z_{m} s_{m}+\frac{\rho}{2}\left(s_{m}-d_{m}\right)^{2}, \\
& \text { if } b_{m}=1: \min _{s_{m}}-\log \left(1-e^{-s_{m}}\right)-z_{m} s_{m}+\frac{\rho}{2}\left(s_{m}-d_{m}\right)^{2} .
\end{aligned}
$$

We note that (21a) is a quadratic minimization. Thus it has a unique solution given by

$$
s_{m}=(1 / \rho)\left(1-z_{m}\right)+d_{m} .
$$

For (21b), the first order optimality condition implies that

$$
\frac{1}{1-e^{s_{m}}}-z_{m}+\rho\left(s_{m}-d_{m}\right)=0
$$

However, (23) is a transcendental equation. For arbitrary choices of $\left(z_{m}, d_{m}, \rho\right)$, there is no closed form solution in general.

To solve for a numerical solution of (23), we construct a nonlinear mapping (for a fixed $\rho$ ) as follows. We first consider the equation

$$
\frac{1}{e^{x}-1}=\rho x-w
$$

where we identify (24) as (23) by noting $x=s_{m}$ and $w=z_{m}+$ $\rho d_{m}$. Let $\left[w_{\min }, w_{\max }\right]$ be the interval in which $w$ is defined, we partition $\left[w_{\min }, w_{\max }\right]$ into $L$ equidistance subintervals and consider a sequence

$$
w_{\ell}=w_{\min }+\ell(\Delta w)
$$

for $\ell=1, \ldots, L$, where $\Delta w=\left(w_{\max }-w_{\min }\right) / L$ is the length of the subinterval. For each $\ell$, we numerically determine the solution of (24) using fzero in MATLAB. This returns a sequence $\left\{x\left(w_{\ell}\right): \ell=1, \ldots, L\right\}$, which are the solutions of (24) for $L$ particular values of $w$. For any other $w \in\left[w_{\min }, w_{\max }\right]$, we interpolate the result using a linear interpolation scheme, given by

$$
x(w)=x\left(w_{\ell}\right)+\left(\frac{x\left(w_{\ell+1}\right)-x\left(w_{\ell}\right)}{w_{\ell+1}-w_{\ell}}\right)\left(w-w_{\ell}\right),
$$

where $\ell$ is chosen such that $w_{\ell} \leq w \leq w_{\ell+1}$.

We note that the computational cost of solving (23) includes the construction of a sequence $\left\{x\left(w_{\ell}\right): \ell=1, \ldots, L\right\}$ and a linear interpolation step. The former can be determined offline because it is independent of the image data. The latter is evaluated using (26), which is a scalar update and can be implemented in parallel.

\section{3. $v$-subproblem}

The $\boldsymbol{v}$-subproblem defined in (14c) is

$$
\underset{\boldsymbol{v}}{\operatorname{minimize}} \lambda\|\boldsymbol{v}\|_{1}-\boldsymbol{r}^{T}(\boldsymbol{v}-\boldsymbol{D} \boldsymbol{c})+\frac{\gamma}{2}\|\boldsymbol{v}-\boldsymbol{D} \boldsymbol{c}\|^{2} .
$$

Applying the shrinkage formula [12], the solution is

$$
\boldsymbol{v}=\max (|\boldsymbol{D} \boldsymbol{c}+\boldsymbol{r} / \gamma|-\lambda / \gamma, 0) \cdot \operatorname{sign}(\boldsymbol{D} \boldsymbol{c}+\boldsymbol{r} / \gamma) .
$$

The overall algorithm is summarized in Algorithm 1.

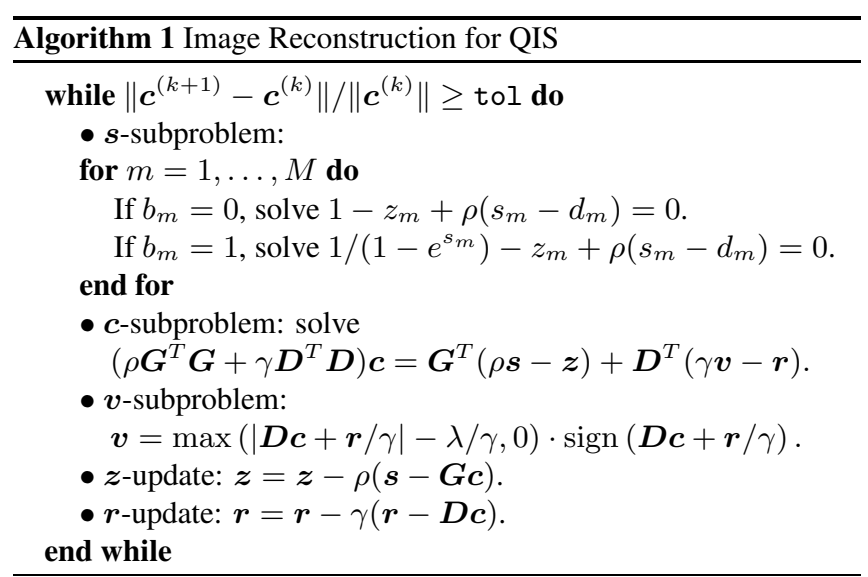




\section{EXPERIMENTAL RESULTS}

\subsection{Convergence to Ideal MLE Solution}

The purpose of the first experiment is to verify the convergence of the proposed algorithm (without the TV penalty) towards the theoretical MLE solution. To this end, we consider the case where the photon count $s_{m}$ is piecewise constant within each subinterval $K n \leq m \leq$ $K(n+1)$ for $n=0, \ldots, N-1$. This implies that the discrete filter $g_{m}$ is a box function of support $[0, K]$. Consequently, the MLE has a closed form solution [10]

$$
\boldsymbol{c}^{(\mathrm{MLE})}=\mathcal{P}_{\mathcal{D}}\left(-\log \left(1-\boldsymbol{G}^{T} \boldsymbol{b} / K^{2}\right)\right)
$$

where $\mathcal{P}_{\mathcal{D}}$ is the projection onto the search domain $\mathcal{D} \stackrel{\text { def }}{=}[0,1]^{N}$.

As a comparison we also consider a gradient descent algorithm presented in [15]. The gradient descent algorithm is applied to solve a simplified case of (11) where there is no total variation penalty (i.e., $\lambda=0$ ). In this case, we substitute the constraint $\boldsymbol{s}=\boldsymbol{G} \boldsymbol{c}$ into the objective of (11) to obtain an unconstrained minimization problem in $\boldsymbol{c}$. We expect the gradient descent iterates to converge to $\boldsymbol{c}^{\text {(MLE) }}$ because the objective $F(c)$ of the unconstrained problem is convex. At the $k$ th iteration, the descent update is given by

$$
\boldsymbol{c}^{(k+1)}=\boldsymbol{c}^{(k)}-\alpha \nabla F\left(\boldsymbol{c}^{(k)}\right) /\left\|\nabla F\left(\boldsymbol{c}^{(k)}\right)\right\|,
$$

where the gradient is (with elementwise multiplication and division)

$$
\nabla F(\boldsymbol{c})=\boldsymbol{G}^{T}\left((\mathbf{1}-\boldsymbol{b})+\boldsymbol{b} \cdot\left(\frac{-e^{-G \boldsymbol{c}}}{1-e^{-G \boldsymbol{c}}}\right)\right),
$$

and the step size $\alpha$ is determined by the standard line search.

Figure 3 shows the mean squared error (MSE) as a function of runtime. It is evident from the figure that the proposed ADMM algorithm has a significantly faster convergence than the descent algorithm. Here, runtime (instead of iteration counts) is considered because the cost per iteration of the descent algorithm is more than that of the ADMM algorithm due to the line search, although the former requires fewer iterations.

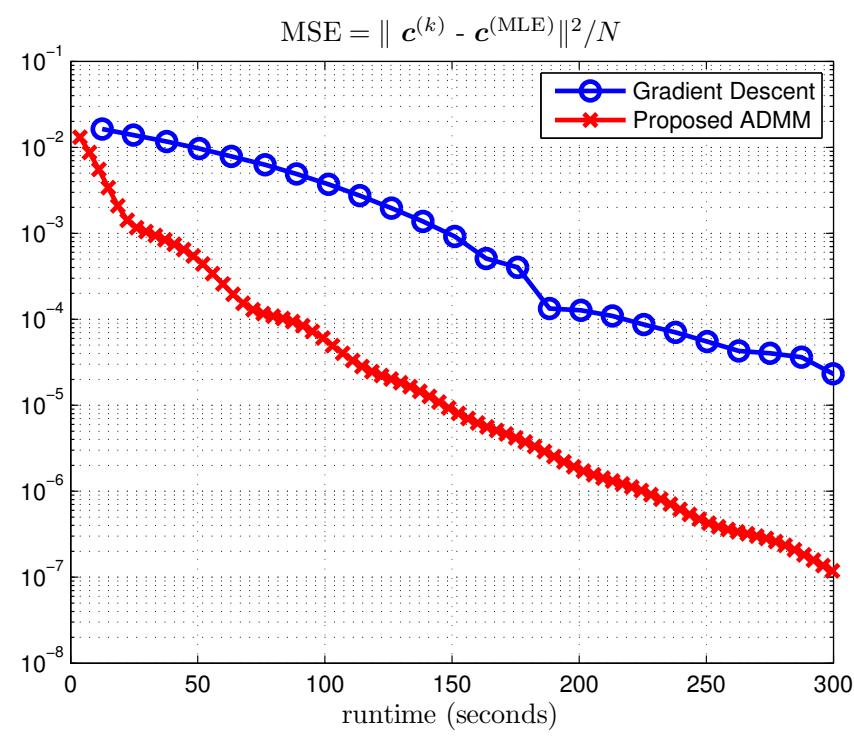

Fig. 3: MSE $\stackrel{\text { def }}{=}\left\|\boldsymbol{c}^{(k)}-\boldsymbol{c}^{(\mathrm{MLE})}\right\|^{2} / N$ as a function of runtime (sec).

\subsection{Reconstruction Quality}

To compare the quality of the reconstructed images, we consider an image of size $100 \times 100$. To simulate a realistic scenario, we define the interpolation kernel $\varphi(x)$ as a Gaussian kernel of size $9 \times 9$ and unit variance. The oversampling ratio is $K=16$, and $\beta(x)$ is a box function of size $K \times K$. Poisson measurements are generated and quantized at a threshold of 1 photon count. The observed binary data is fed into the proposed algorithm, and results are obtained when the algorithm terminates.

The internal parameters of the algorithm are defined as follows. We set $\lambda=10$ to put moderate strength of the total variation penalty. The quadratic penalty parameters are set to $\rho=10$ and $\gamma=1$. We stop the iteration of the algorithm when either the number of iterations exceeds a maximum of 100, or the relative change satisfies $\left\|\boldsymbol{c}^{(k+1)}-\boldsymbol{c}^{(k)}\right\| /\left\|\boldsymbol{c}^{(k)}\right\| \leq 10^{-3}$.

Figure 4 shows the results of the reconstruction. In Figure 4(a), we show the reconstructed result by running the proposed algorithm without total variation (i.e., $\lambda=0$ ). As illustrated in the previous experiment, this result converges to the ideal MLE solution as we increase the iteration number. For this particular image, the MLE solution achieves a PSNR of $25.62 \mathrm{~dB}$. In Figure 4(b), we show the result when $\lambda=10$. This corresponds to the MAP solution with a total variation prior. Evidently, the PSNR value increases to 30.79 $\mathrm{dB}$, which is significantly higher than that of the MLE solution. In addition, we remark from Figure 3 that the runtime of the proposed ADMM algorithm is much shorter than that of the descent algorithm. These results indicate that the proposed scheme achieves better reconstruction quality with less computation.

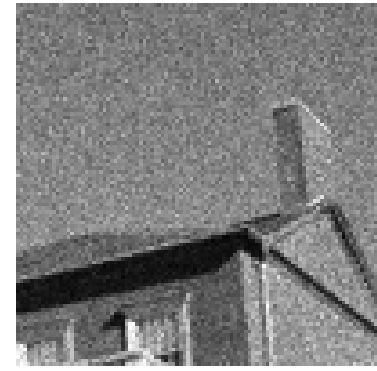

(a) MLE Solution [15] $\lambda=0$

$\mathrm{PSNR}=25.62 \mathrm{~dB}$

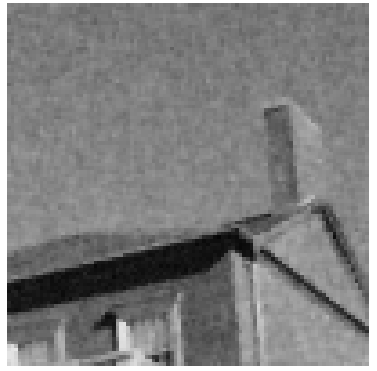

(b) MAP Solution (proposed)

$$
\lambda=10
$$

$\mathrm{PSNR}=30.79 \mathrm{~dB}$
Fig. 4: Comparison of the proposed algorithm when total variation penalty is switched on/off. In both cases, we set $K=16$.

\section{CONCLUSION}

A fast image reconstruction algorithm for quantum image sensors is proposed. The new algorithm is based on the concept of alternating direction method of multipliers (ADMM). We presented fast solutions to each subproblem associated with the ADMM algorithm. These include a polyphase-domain filtering to achieve efficient matrix inversion, and a nonlinear mapping to find solutions of a transcendental equation. The new algorithm demonstrates superior performance and better convergence speed than the standard gradient descent algorithm. Future work will be focused on the analysis of solutions in the large-scale limits. 


\section{REFERENCES}

[1] M. A. Itzler, S. Cova, M. Wahl, and A. Tomita, "Introduction to the issue on single photon counting: Detectors and applications," IEEE J. Sel. Topics Quantum Electron., vol. 13, no. 4, pp. 849-851, Aug. 2007.

[2] W. H. P. Pernice, C. Schuck, O. Minaeva, M. Li, G. N. Goltsman, A. V. Sergienko, and H. X. Tang, "High-speed and highefficiency travelling wave single-photon detectors embedded in nanophotonic circuits," Nature Communications, vol. 3, pp. 1325, Dec. 2012.

[3] E. R. Fossum, "The Quantum Image Sensor (QIS): Concepts and challenges," in Proc. OSA Topical Mtg Computational Optical Sensing and Imaging, Jul 2011, Paper JTuE1.

[4] D. E. Schwartz, E. Charbon, and K. L. Shepard, "A singlephoton avalanche diode array for fluorescence lifetime imaging microscopy," IEEE J. Solid-State Circuits, vol. 43, no. 11, pp. 2546-2557, Nov. 2008.

[5] C. Niclass, A. Rochas, P. A. Besse, and E. Charbon, "Design and characterization of a CMOS 3-D image sensor based on single photon avalanche diodes," IEEE J. Solid-State Circuits, vol. 40, no. 9, pp. 1847-1854, Sep. 2005.

[6] A. McCarthy, R. J. Collins, N. J. Krichel, V. Fernández, A. M. Wallace, and G. S. Buller, "Long-range time-of-flight scanning sensor based on high-speed time-correlated single-photon counting," Applied Optics, vol. 48, no. 32, pp. 6241-6251, Nov. 2009.

[7] B. S. Robinson, A. J. Kerman, E. A. Dauler, R. J. Barron, D. O. Caplan, M. L. Stevens, J. J. Carney, S. A. Hamilton, J. K. Yang, and K. K. Berggren, "781 Mbit/s photon-counting optical communications using a superconducting nanowire detector," $O p$ tics Letters, vol. 31, no. 4, pp. 444-446, 2006.
[8] F. Yang, L. Sbaiz, E. Charbon, S. Süsstrunk, and M. Vetterli, "Image reconstruction in the gigavision camera," in Proc. Intl' Conf. Computer Vision Workshops (OMNIVIS), 2009, pp. 2212-2219.

[9] M. Unser and M. Eden, "Maximum likelihood estimation of linear signal parameters for Poisson processes," IEEE Trans. Acoust., Speech, Signal Process., vol. 36, no. 6, pp. 942-945, Jun. 1988.

[10] F. Yang, Y. M. Lu, L. Sbaiz, and M. Vetterli, "Bits from photons: Oversampled image acquisition using binary poisson statistics," IEEE Trans. Image Process., vol. 21, no. 4, pp. 1421-1436, Apr. 2012.

[11] J. Zhang, F. Yang, T. Vogelsang, D. G. Stork, and M. Vetterli, "Multichannel sampling of low light level scenes wth unknown shifts," in Proc. Intl' Conf. Image Process., 2013, pp. 863-867.

[12] S. H. Chan, R. Khoshabeh, K. B. Gibson, P. E. Gill, and T. Q. Nguyen, "An augmented Lagrangian method for total variation video restoration," IEEE Trans. Image Process., vol. 20, no. 11, pp. 3097-3111, Nov. 2011.

[13] S. Boyd, N. Parikh, E. Chu, B. Peleato, and J. Eckstein, "Distributed optimization and statistical learning via the alternating direction method of multipliers," Found. Trends Mach. Learn., vol. 3, no. 1, pp. 1-122, Jan. 2011.

[14] P. P. Vaidyanathan, Multirate Systems and Filter Banks, Prentice Hall, 1992.

[15] F. Yang, Y. M. Lu, L. Sbaiz, and M. Vetterli, "An optimal algorithm for reconstructing images from binary measurements," in Proc. IS\&T/SPIE Conf. Computational Imaging VIII, 2010, vol. 7533 , paper $75330 \mathrm{~K}$. 